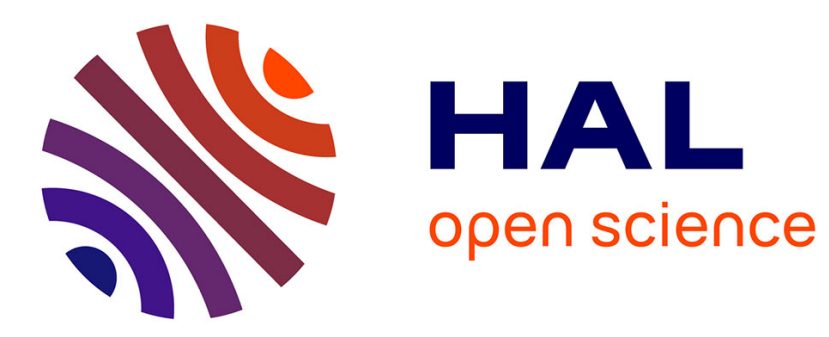

\title{
Modulations liées au mouvement d'un jet moléculaire
} M. Annabi, D. Gillet

\section{To cite this version:}

M. Annabi, D. Gillet. Modulations liées au mouvement d'un jet moléculaire. Revue de Physique Appliquée, 1973, 8 (1), pp.1-6. 10.1051/rphysap:01973008010100 . jpa-00243650

\section{HAL Id: jpa-00243650 https://hal.science/jpa-00243650}

Submitted on 1 Jan 1973

HAL is a multi-disciplinary open access archive for the deposit and dissemination of scientific research documents, whether they are published or not. The documents may come from teaching and research institutions in France or abroad, or from public or private research centers.
L'archive ouverte pluridisciplinaire HAL, est destinée au dépôt et à la diffusion de documents scientifiques de niveau recherche, publiés ou non, émanant des établissements d'enseignement et de recherche français ou étrangers, des laboratoires publics ou privés. 


\title{
REVUE DE PHYSIQUE APPLIQUÉE
}

\author{
Supplément au «Journal de Physique "
}

Classification

Physics abstracts

$13.00-13.30-05.00-05.20$

\section{MODULATIONS LIÉES AU MOUVEMENT D'UN JET MOLÉCULAIRE}

\author{
M. ANNABI et D. GILLET \\ Laboratoire de Spectroscopie Hertzienne et d'Electronique, Besançon
}

(Reçu le 23 juin 1972)

\begin{abstract}
Résumé. - Un dispositif est décrit, qui permet d'obtenir la distribution des vitesses dans un jet moléculaire. Pour ce faire, on utilise une modulation spatiale du champ de radiofréquence induisant les transitions qui produit des bandes latérales dépendant de la vitesse des molécules. En outre, on décrit un autre dispositif qui permet d'obtenir une rotation spatiale du champ de radiofréquence et qui conduit à un déplacement des composantes du spectre Zeeman ; ce déplacement dépend du signe du facteur $g$ moléculaire.

Abstract. - In this paper we describe a device to obtain the velocity distribution in a molecular beam. For this purpose we use a space modulation of the radiofrequency field inducing molecular transitions which produces side-bands depending on the velocity of the molecules. Following the same principle, we describe a device giving a spatial rotation of the radiofrequency field giving a shift of the components of the Zeeman pattern depending on the sign of the $g$ factor.
\end{abstract}

1. Introduction. - Les dispositifs de modulation spatiotemporelle du champ d'irradiation décrits ici sont utilisés dans un spectromètre à jet moléculaire dont le principe est le suivant [1] : un jet de molécules (formol dans le cas de nos expériences) issu d'une source traverse un focaliseur électrostatique permettant d'effectuer un tri des molécules en fonction de leur état quantique, les molécules peuplant l'état supérieur d'énergie des doublets $\mathrm{K}$ étant focalisées sur l'axe. Le jet traverse ensuite une région d'irradiation où il est soumis à un champ radiofréquence de fréquence variable. Un certain nombre de molécules passe dans l'état inférieur d'énergie. Un second focaliseur identique au premier élimine les molécules qui ont subi la transition et le passage à la résonance se traduit par un appauvrissement du jet que l'on détecte à l'aide d'une jauge à ionisation.

La région d'irradiation peut être simple ou double [2] Dans le cas présent, la deuxième zone d'irradiation est formée par un condensateur aux plateaux duquel on applique une tension alternative à une fréquence correspondant au doublet $\mathrm{K}$ étudié et d'amplitude permettant la saturation de cette transition. Ce condensateur permet d'étudier les effets d'une quelconque première zone d'irradiation [2] qui peut être un montage de passage adiabatique [3] ou un dispositif de modulation du champ radiofréquence irradiant le jet.

2. Etude, par modulation, des vitesses dans un jet moléculaire. - L'un des problèmes que nous avons essayé de résoudre est l'étude de la répartition des vitesses dans le spectromètre à jet à deux focaliseurs. Une des méthodes générales utilisées antérieurement pour étudier les vitesses dans un jet consiste à moduler mécaniquement le jet [4], [5], [6] en l'interrompant par des disques fendus et en détectant les variations d'intensité ainsi produites. Une autre méthode utilise la chute des particules dans le champ de la pesanteur [7] ou des champs inhomogènes [8]. La méthode que nous proposons ici utilise l'intermédiaire d'un état quantique donné et non plus l'ensemble du jet ; de plus, la modulation est produite sur le champ électromagnétique induisant les transitions par le mouvement même des molécules.

A cette fin, on fait passer le jet dans un dispositif donnant une modulation spatiale périodique de l'amplitude du champ. La vitesse de chaque molécule transforme cette modulation spatiale en une modulation dans le temps dépendant de la vitesse de chaque molécule. Le dispositif utilisé consiste en une série de fils tendus perpendiculairement à la direction du jet, les uns sont mis à la masse et les autres soumis à la tension radiofréquence (Fig. 1). On voit qu'entre 2 fils (points A) le module du champ est maximum et qu'il est nul en B. L'allure du champ radiofréquence en fonction du temps est représentée sur la figure $\overline{1} 1 b$. La phase du champ variant de $\pi$ entre 2 paires de fils successives, la période spatiale est $2 d$.

Le champ vu par une molécule s'écrit alors $\mathcal{E}=f(t) \sin \omega t, f(t)$ étant une fonction périodique du 
temps par suite du mouvement de la molécule; on écrira :

$$
f(t)=\sum_{0}^{\infty} a_{n} \sin \left(n \Omega t+\varphi_{n}\right), \quad \Omega=2 \pi \frac{v}{2 d} .
$$

En se limitant aux premiers termes on écrira :

$$
\begin{aligned}
\mathcal{E}=\mathcal{E}_{0} \sin \omega t+\mathcal{E}_{1} \cos [ & \left.(\omega-\Omega) t-\varphi_{1}\right]- \\
& -\mathcal{E}_{1} \cos \left[(\omega+\Omega) t+\varphi_{1}\right]+\cdots
\end{aligned}
$$

avec

$$
a_{0}=\varepsilon_{0}=\frac{1}{T} \int_{0}^{T} f(t) \mathrm{d} t, \text { etc... }
$$
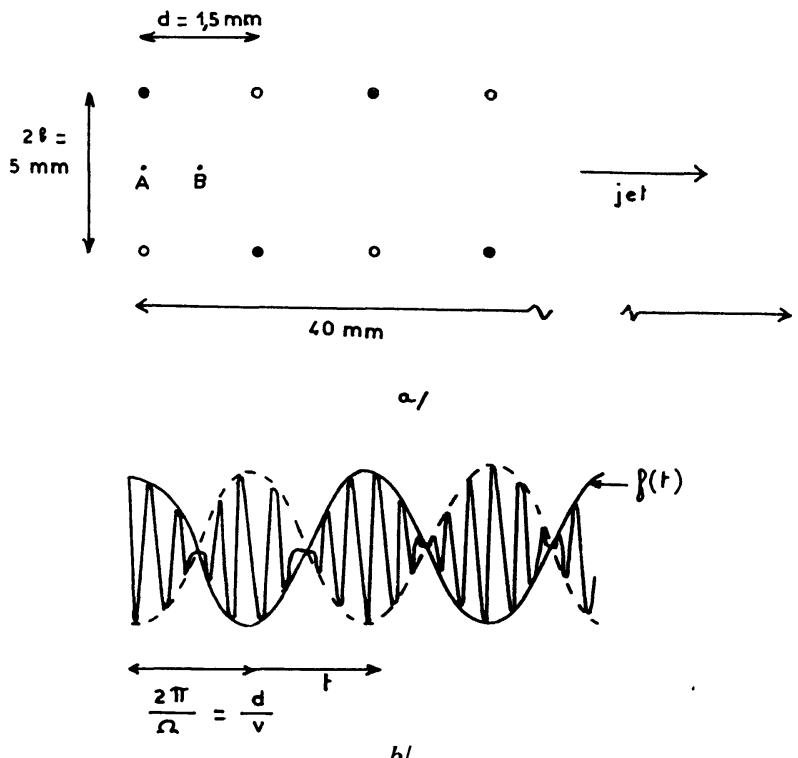

b/

FIG. 1. - Modulation spatiale du champ radiofréquence.

2.1 Allure Des SIgNauX ObSERvés. - La forme de la fonction $f(t)$ peut se calculer théoriquement à partir de la relation 135 bis de la référence [9]. On trouve, sur l'axe du spectromètre, en prenant $\delta_{1}=1$ :

$$
\varepsilon_{2}=0, \varepsilon_{0}=0, \varepsilon_{3}=\frac{1}{4 \operatorname{sh}^{2}\left(\frac{\pi b}{d}\right)} \not 10^{-4} .
$$

$\varepsilon_{0}$ est différent de zéro en dehors de l'axe, un ordre de grandeur en est 2 th $(\pi z / d), z$ étant la distance à l'axe. Le champ d'irradiation se compose donc pour les molécules se déplaçant sur l'axe de bandes latérales $\omega \pm n \Omega$. Les molécules se déplaçant en dehors de l'axe verront en plus une "porteuse " centrale de fréquence $\omega / 2 \pi$.

Le signal observé se composera donc d'une raie centrale lorsque $\omega=\omega_{\mathbf{K}}$, et qui aura l'allure de la raie de résonance habituelle $\left(\omega_{\mathrm{K}} / 2 \pi\right.$ étant la fréquence correspondant à l'intervalle du doublet $\mathrm{K}$ étudié). De plus, il apparaîtra d'autres raies latérales situées à $\pm n \Omega$ dont les 2 premières seules $(n=1)$ seront visibles. Comme il existe une répartition des vitesses dans le jet, le mouvement transforme cette dernière en une répartition des fréquences et elle interviendra dans la forme des raies latérales.
2.2 Calcul De LA Forme Des Signaux. - On se trouve en présence d'un système quantique, présentant 2 niveaux d'énergie, et irradié par 3 champs électriques radiofréquence :

$\varepsilon_{0}$ de fréquence $\omega / 2 \pi$;

$\varepsilon_{1}^{\prime}$ de fréquence $\omega+\Omega / 2 \pi$;

et $\varepsilon_{1}^{\prime \prime}$ de fréquence $\omega-\Omega / 2 \pi$.

En se limitant aux transitions à un photon et en négligeant le recouvrement des raies dues à chacun des trois champs, la probabilité de transition est donnée par [10] :

$$
\frac{4 K^{2}}{\gamma^{2}} \sin ^{2}\left(\frac{\gamma t}{2}\right)=p
$$

pour le champ $\varepsilon_{0}$, où $K_{0}$ est l'élément de matrice de la perturbation correspondant au champ $\varepsilon_{0}$, et

$$
\gamma=\left[\left(\omega_{\mathrm{K}}-\omega\right)^{2}+4 K_{0}^{2}\right]^{1 / 2}
$$

$\left(\omega_{K / 2 \pi}\right)$ étant la fréquence correspondant à l'intervalle du doublet $\mathrm{K}$ entre les niveaux duquel sont induites les transitions. Pour les raies latérales, on obtient des expressions semblables.

Tenons compte de la répartition des vitesses. Pour la raie centrale, le signal vaut $\int_{0}^{\infty} p r(v) \mathrm{d} v$, compte tenu de la répartition $r(v)$. Il n'en est plus de même pour les raies latérales, à cause de la répartition des fréquences de résonance. Chacune de ces raies est formée par la superposition des signaux donnés par les différentes molécules de vitesses comprises entre $v$ et $v+\mathrm{d} v$. Une molécule de vitesse $v$ donne un signal (pour une raie latérale) :

$$
\begin{aligned}
& \frac{4 K_{1}^{2}}{\left(\omega_{\mathrm{K}}-\omega+\frac{2 \pi v}{2 d}\right)^{2}+4 K_{1}^{2}} \times \\
& \quad \times \sin ^{2}\left\{\left[\left(\omega_{\mathrm{K}}-\omega+\frac{2 \pi v}{2 d}\right)^{2}+4 K_{1}^{2}\right]^{1 / 2} \frac{l}{v}\right\}
\end{aligned}
$$

$l$ étant la longueur de la zone d'irradiation $(4 \mathrm{~cm})$; la résonance de cette molécule étant centrée en

$$
\omega=\omega_{\mathrm{K}}+\frac{2 \pi v}{2 d}
$$

(fréquence affichée au générateur). Le nombre de molécules résonnant à cette fréquence est le nombre de molécules compris entre $v$ et $v+\mathrm{d} v$, soit $r(v) \mathrm{d} v$ et le signal complet composant une raie latérale est :

$$
\begin{aligned}
S= & \int_{0}^{\infty} \frac{4 K_{1}^{2}}{\left(\omega_{\mathrm{K}}-\omega+\frac{2 \pi v}{2 d}\right)^{2}+4 K_{1}^{2}} \times \\
& \times \sin ^{2}\left\{\left[\left(\omega_{\mathrm{K}}-\omega+\frac{2 \pi v}{2 d}\right)^{2}+4 K_{1}^{2}\right]^{1 / 2} \frac{l}{v}\right\} r(v) \mathrm{d} v .
\end{aligned}
$$

Le calcul numérique de cette intégrale pour différentes valeurs du champ d'irradiation a été fait à la 
machine. Les niveaux de la molécule de formol étant dégénérés, nous avons admis que le signal était obtenu en additionnant les probabilités des transitions $\Delta M=0, \Delta M= \pm 1$ avec des poids égaux. Nous appuyant sur un résultat expérimental de D. Gillet [11] nous supposons qu'à la sortie du premier focaliseur, les molécules se répartissent également entre les divers états $M$ possibles.

Nous avons tracé figure 2 les courbes donnant $S=f(\omega)$ pour le modèle de répartition :

$$
r_{N}(v) \alpha V^{N} \exp \left(-\frac{v^{2}}{\alpha^{2}}\right)
$$

avec $N=3$. $\alpha$ est la constante rencontrée lors des études statistiques sur les gaz.
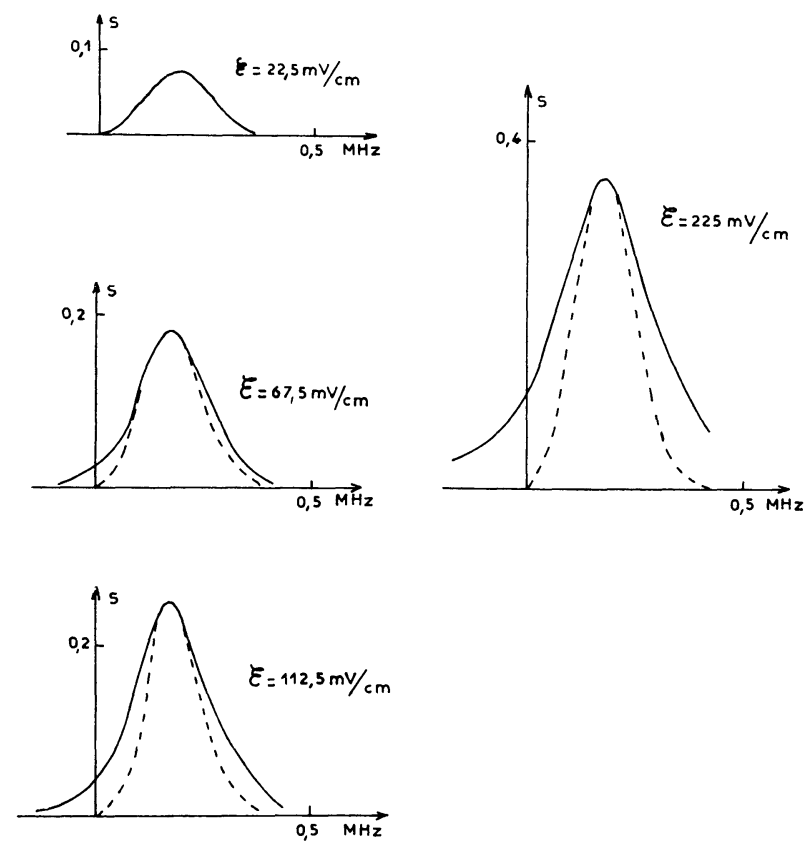

Fig. 2. - Probabilité de transition pour la composante supérieure du spectre en fonction de l'écart à la fréquence de résonance naturelle.

$$
\text { - En trait plein } S \text {. }
$$$$
\text { - En pointillé } r_{3}(v) \text {. }
$$

La fonction $r(v)$ est normalisée pour le calcul numérique de $S$. Cette fonction $r(v)$ peut se justifier pour des sources fonctionnant par effusion [10], [12] et pour des distances de quelques dizaines de centimètres entre les 2 focaliseurs [12]. On voit que la forme des raies latérales reproduit la courbe $r(v)$ (tracée en pointillés après coïncidence des ordonnées des maximums), tout au moins aux bas niveaux d'irradiation. Lorsque le champ d'irradiation augmente, des différences apparaissent entre $r(v)$ et $S$, la largeur de cette dernière courbe augmentant. Cette situation est analogue à celle qui se produit par exemple en résonance magnétique des raies inhomogènes [13] où la raie de résonance reproduit en champ bas la fonction de répartition lorsque la largeur d'une raie individuelle est plus petite que la largeur de la fonction de répartition, mais s'élargit ensuite si le champ d'irradiation augmente. On voit donc que la détermination expérimentale de $r(v)$ sera d'autant meilleure que le champ d'irradiation sera plus faible. Nous avons étudié de même la répartition $r(v)$ avec $N=1$ qu'on obtient pour des focaliseurs très rapprochés [11].

2. 3 COMPARAISON AVEC L'EXPÉRIENCE. - La figure 3 reproduit le spectre obtenu pour la transition $J_{K}=4_{3}$ du formol, pour un faible niveau d'irradiation.

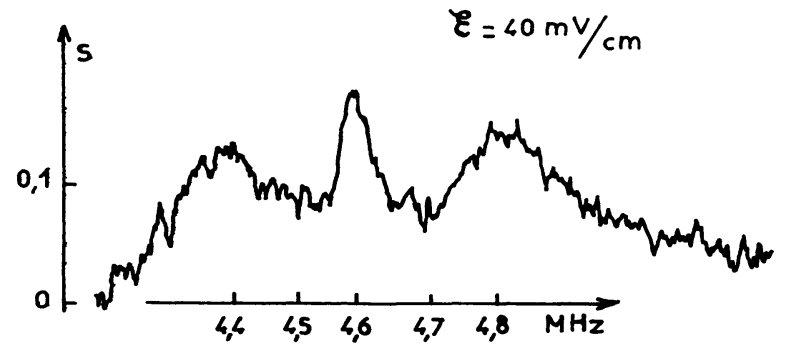

FrG. 3. - Spectre expérimental : transition $4_{3}$ du formol.

On observe expérimentalement que les bandes latérales présentent 2 maximums situés à environ $\pm 200 \mathrm{kHz}$ de part et d'autre de la raie principale, alors que les positions théoriques des maximums sont 110 et $190 \mathrm{kHz}$ pour respectivement $r_{N=1}(v)$ et $r_{N=3}(v)$. La figure 4 montre la reproduction d'une moitié du spectre expérimental en fonction de l'écart à la résonance ; on y a aussi tracé les courbes $S$ correspondant aux 2 répartitions proposées.

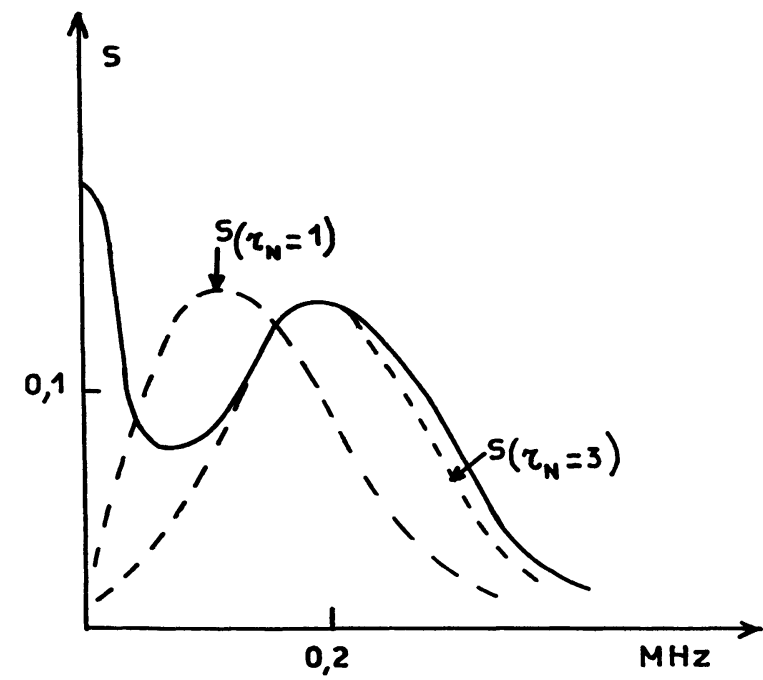

FIG. 4. - Comparaison du spectre expérimental avec les formes de raies théoriques.

On voit que les résultats expérimentaux semblent justifier une répartition assez voisine de $r_{N=3}$ avec toutefois des vitesses un peu supérieures. (Vitesse la plus probable $600 \mathrm{~m} / \mathrm{s}$ au lieu de $570 \mathrm{~m} / \mathrm{s}$.) On peut expliquer ce résultat en remarquant que les interactions dans le jet [1] (qui sont d'ailleurs difficiles à évaluer) tendent à décomposer ce dernier et à favoriser les molécules les plus rapides. 
Notons que les résultats trouvés sont en accord avec les méthodes de saturation et d'effet Zeeman-Stark relativiste [12].

2.4 REMARQUE. - On peut utiliser les expériences de saturation pour calibrer le champ de radio fréquence :

1) A partir des courbes (Fig. 2) on peut évaluer les probabilités de transition théoriques pour les maximums des raies latérales;

2) Les ordonnées des courbes expérimentales ne sont pas arbitraires. En effet, on sait que si le champ d'irradiation est grand, la probabilité de transition tend vers 0,5 [10] d'où un moyen d'étalonner les spectres en ordonnées.

En utilisant les pentes à l'origine des courbes de saturation on trouve $\varepsilon_{1}=18 \mathrm{mV} / \mathrm{cm}$ pour une tension d'irradiation de $10 \mathrm{mV}$.

On peut calibrer de même la raie centrale, mais seulement de façon moins absolue; la composante centrale du spectre n'existe comme nous l'avons dit qu'à cause des molécules ne se déplaçant pas sur l'axe du spectromètre. Une valeur approximative est $\varepsilon_{0}=3,8 \mathrm{mV} / \mathrm{cm}$ notablement inférieure à $\delta_{1}$ ce qui montre que la plupart des molécules sont bien concentrées sur l'axe. Le fait que la raie de résonance centrale soit plus grande que les bandes latérales ne doit pas étonner bien que $\varepsilon_{0}<\varepsilon_{1}$. En effet, la raie centrale qui est une raie de résonance ordinaire apparaît pour des tensions d'irradiation très faibles [1], [10].

3. Détermination du signe du facteur $g$ moléculaire. - Les mesures d'effet Zeeman en champ radiofréquence linéaire et homogène permettent de déterminer le facteur $g$ moléculaire en valeur absolue. En appliquant un champ tournant [10] on peut déterminer le signe du facteur $g$ d'une particule [14]. Cette méthode présente toutefois certains inconvénients (en particulier elle nécessite la réalisation d'un déphaseur radiofréquence parfaitement équilibré pour chaque fréquence étudiée). Aussi avons-nous pensé arriver au résultat en faisant traverser au jet une zone d'irradiation où l'orientation du champ radiofréquence varie dans l'espace. Cette expérience est directement inspirée par la précédente.

3.1 Principe. - Sous l'effet d'un champ magnétique continu extérieur, chacun des deux niveaux supérieur et inférieur d'un doublet (J, K) de la molécule de formol est décomposé en $2 \mathrm{~J}+1$ niveaux, d'énergie :

$$
W=-g_{\mathrm{i}, \mathrm{s}} \beta_{1} H M
$$

$g_{\mathrm{i}, \mathrm{s}}$ est le facteur $g$ de l'état supérieur ou inférieur; $\beta_{1}$ le magnéton nucléaire ;

$H$ le champ magnétique extérieur ; $M$ le nombre Zeeman.
On néglige la structure hyperfine, non résolue.

Un champ électrique $\boldsymbol{\delta}$ radiofréquence perpendiculaire à $H$ induit des transitions $\Delta M=+1$ où $\Delta M=-1$.

Les transitions

$$
|\mathrm{s}, M>\rightarrow| \mathrm{i}, M-1>
$$

sont induites par la composante circulaire de $\boldsymbol{\delta}$ tournant dans le sens direct $\sigma_{+}$(Fig. 5).

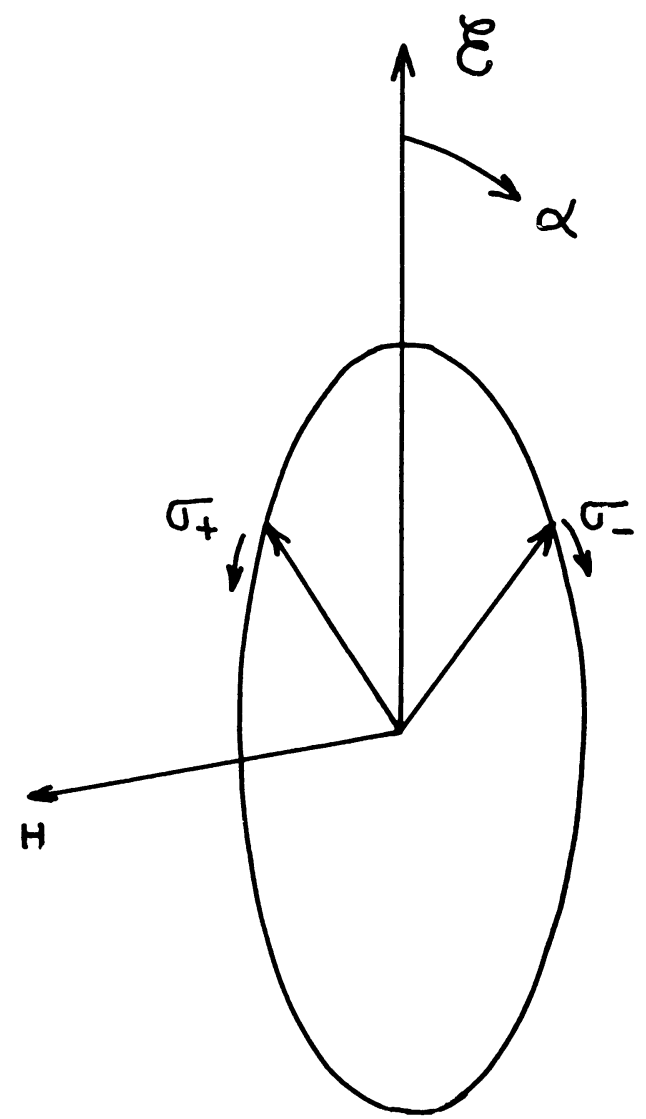

FIG. 5. - Composantes du champ radiofréquence induisant les 2 raies Zeeman.

Elles se produisent à la fréquence :

$$
v_{+}=v_{\mathrm{K}}-g_{\mathrm{s}} M+g_{\mathrm{i}}(M-1) .
$$

Les transitions

$$
|\mathrm{s}, M-1>\rightarrow| \mathrm{i}, M>
$$

sont induites par la composante circulaire tournant dans le sens rétrograde. $\sigma_{-}$, et se produisent à la fréquence :

$$
v_{-}=v_{\mathrm{K}}-g_{\mathrm{s}}(M-1)+g_{\mathrm{i}}(M) .
$$

Supposons qu'au cours de son trajet, le jet moléculaire voie un champ dont l'orientation tourne uniformément dans le temps, par exemple dans le sens rétrograde. Cela revient à dire que $\sigma_{+}$est ralenti, $\sigma_{-}$accéléré, les fréquences angulaires devenant respectivement $v-\alpha$ et $v+\alpha$ autrement dit la résonance $v_{+}$se produira 
pour une fréquence affichée au générateur supérieure à la valeur donnée plus haut, et $v_{-}$à une fréquence affichée inférieure.

Supposons $g$ négatif, alors :

$$
v_{+}>v_{\mathrm{K}} \text { et } v_{-}<v_{\mathrm{K}} \text {. }
$$

L'écart des deux raies sera plus grand que celui observé en champ $\delta$ homogène. Si on inverse le sens du champ magnétique, l'écart des deux raies sera plus petit.

Si $g>0$, les conclusions sont inversées.

3.2 EXPÉRIENCE. - La zone d'irradiation du jet est formée par une hélice double (dans notre cas à gauche pour un observateur regardant dans le sens du jet) dont l'un des brins est à la masse et l'autre soumis à la tension radiofréquence (Fig. 6).

Le champ magnétique est parallèle au jet.

On voit que le vecteur champ radiofréquence tourne au fur et à mesure du déplacement d'une molécule de vitesse $v$. Si $d$ est le pas de l'hélice, $\alpha=v / d$.

Pour $d=2 \mathrm{~cm}, v=550 \mathrm{~m} / \mathrm{s}$ on trouve $\alpha=27,5 \mathrm{kHz}$.

La figure 7 montre les résultats obtenus pour la transition $4_{31}-4_{32} \mathrm{du}$ formol $\left(v_{\mathrm{K}}=4,574 \mathrm{MHz}\right)$. Ces déplacements correspondent à une fréquence angulaire $\alpha$ comprise entre 25 et $30 \mathrm{kHz}$. On voit que

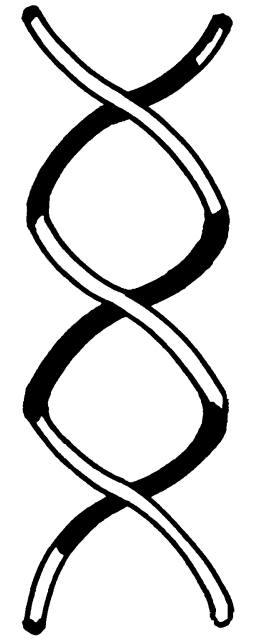

Fig. 6. - Hélice double produisant un champ de radiofréquence dont l'orientation tourne dans l'espace.

les effets sont ceux qui se produisent si $g<0$. On trouve de même $g<0$ pour les raies $13_{5}$ et $5_{3}$. C'est, à notre connaissance, la première détermination expérimentale directe du signe du facteur $g$ de ces états du formol, qui n'avait été déterminé précédemment [15] que par des considérations théoriques.
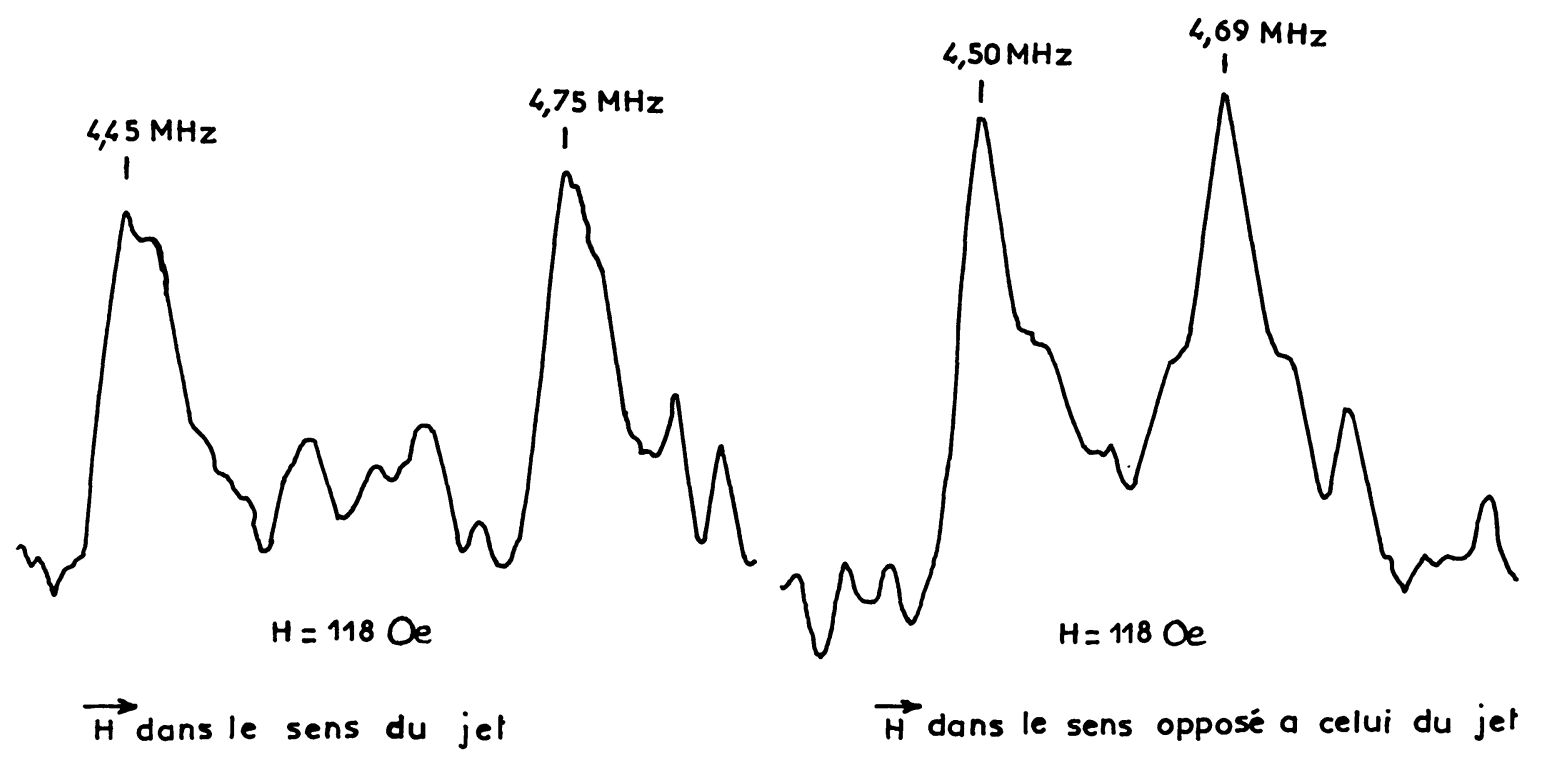

FIG. 7. - Spectres d'effet Zeeman obtenus à l'aide de l'hélice double.

4. Conclusion. - Nous avons décrit 2 dispositifs dans lesquels on utilise le mouvement même des molécules pour obtenir des effets qui sont produits habituellement en faisant appel à des dispositifs électroniques (modulations, champ tournant). Le fait d'utiliser le mouvement même des molécules permet une approche nouvelle, croyons-nous, de l'étude du mouvement de ces molécules. Le premier dispositif nous a permis de montrer par exemple que dans une expérience de passage adiabatique complet, la répartition des vitesses n'était pas modifiée de façon sen- sible [16] la répartition des vitesses déterminée ainsi peut être utilisée dans le cas [16] où le passage adiabatique n'est pas parfait pour rendre compte du défaut d'adiabaticité. Le deuxième montage permet la détermination du signe du facteur $g$ d'une particule sans dispositif électronique notable produisant un champ tournant. On pourrait également l'utiliser pour étudier la répartition des vitesses mais avec des résultats moins précis par suite du pas assez grand de l'hélice et qu'il est difficile de réduire. Les deux montages sont applicables en principe à tous les types de jets moléculaires. 


\section{Bibliographie}

[1] Genty C., Thèse Besançon, 1970, AO 3676.

[2] Mandret G., C. R. Acad. Sci. Paris 271 (1970) 480 ; Thèse Besançon, 1970, AO 4679.

[3] Annabi M., Chardon J. C., Gillet D., Theobald J. G., C. R. Acad. Sci. Paris 272 (1971) 376.

Annabi M., Chardon J. C., Gillet D., C. R. Acad. Sci. Paris 274 (1972) 544.

Annabi M., Gillet D., C. R. Acad. Sci. Paris 274 (1972) 711.

[4] Expériences de Stern, 1920, voir Bruhat, Thermodynamique, Masson, Paris, 1962.

[5] Bennet A. I. et Esterman I., Distribution of Velocities in Molecular Beams of Potassium. Doct. Diss. Carnegie Institute of Technology, 1953.

[6] Berthier J. P., Constans A., Daury C. et Lostis P., Revue Phys. Appl. 6 (1971) 433.

[7] Esterman I., Simpson O. C., Stern O., Phys. Rev. 71 (1947) 238.
[8] Tang S. Y., Marcus A. B., Muschlitz jr E. E., J. Chem. Phys. 56 (1972) 566.

[9] Durand E., Electrostatique, 1964, t. I, Masson, Paris.

[10] Ramsey N. F., Mol. Beams, 1956, Oxford.

[11] Gillet D., Thèse Besançon, 1970, AO 4680.

[12] Chardon J. C., Gillet D., Luneau S., Theobald J. G., C. R. Acad. Sci. Paris 274 (1972) 495.

[13] a) Portis A. N., Phys. Rev. 91 (1953) 1071 et Magnetic resonance in systems with spectral distribution, Sarah Melon Scaife Radiation Laboratory Pittsburg, 1955.

b) Gabillard R., Grivet P., La Résonance Magnétique nucléaire, CNRS Paris, 1955.

[14] Annabi M., Gillet D., Luneau S., Theobald J. G., C. R. Acad. Sci. Paris 272 (1971) 1256.

[15] Kondo K., Hirakawa H., Miyahara A., OKa J., Shimoda K., J. Phys. Soc. Jap. 15 (1960), 303 et Flygare W., J. Chem. Phys. 42 (1962) 1563.

[16] Annabi M., Thèse Besançon (à paraître), AO 7237. 